\title{
Tratamento endoscópico das lesões biliares
}

\section{Endoscopic treatment of the biliary injuries}

\author{
Everson L. A. Artifon, TCBC-SP1; DéCio Sampalo Couto Júnior, AsCBC-CE²; Paulo Sakal ${ }^{3}$
}

\section{R E S U M O}

\begin{abstract}
As técnicas cirúrgicas convencionais ofertam uma apropriada condição de cura na maioria dos pacientes com estreitamento biliar benigno. Nesta condição, no entanto, o reparo cirúrgico está associado com recorrência tardia da re-estenose em $10 \%$ a $30 \%$ dos pacientes. Neste contexto, os avanços tecnológicos na endoscopia terapêutica promoveram a possibilidade alternativa do tratamento efetivo destas obstruções benignas. Considerações em relação ao tempo de reospitalização e de procedimentos devem ser averiguados em detalhes e ponderados em relação á cirurgia. Estenoses malignas estão relacionadas á colangite, icterícia e dor e, consequentemente, com as alterações sistêmicas relacionadas com a sepsis biliar. A conduta endoscópica cria uma derivação do suco biliar para o duodeno, sendo uma verdadeira derivação biliodigestiva endoscópica e utilizando-se próteses plásticas ou metálicas. O propósito desta revisão é ofertar aos leitores a eficácia do tratamento endoscópico na estenose benigna e maligna biliopancreática.
\end{abstract}

Descritores: Ducto biliar. Trato biliar extra-hepático. Stents.

\section{INTRODUÇÃO}

O desenvolvimento da tecnologia endoscópica e da radiologia intervencionista nas últimas duas décadas tem oferecido uma opção segura à terapêutica cirúrgica das lesões das vias biliares. As estenoses e fístulas são mais freqüentes no contexto pós-operatório, em pacientes jovens e na população ativa. Em muitos casos a cirurgia oferece o tratamento definitivo com intenção paliativa ou terapêutica, contudo não é isenta de complicações'.

A terapêutica endoscópica contribui pela documentação imagenológica, diagnóstico histopatológico e intervenção definitiva, com resultados similares e em alguns casos melhores que o tratamento cirúrgico.

A disponibilidade da intervenção endoscópica ou radiologia intervencionista, na drenagem biliar de etiologia benigna ou maligna, permite preparar o paciente para cirurgia ou mesmo paliação definitiva.

Dentre as estenoses biliares, incluiremos neste capítulo as de etiologias benignas e malignas, além de comentários em relação a aspectos técnicos direcionados ao objetivo comum do tratamento endoscópico e radiológicointervencionista: a drenagem biliar.

\section{ESTENOSES BENIGNAS}

\subsection{Etiologia e Diagnóstico}

As principais causas de estenoses benignas são decorrentes de procedimentos cirúrgicos (iatrogênica ou anastomoses difíceis) e colangite esclerosante primária (Ta- bela 1), com o diagnóstico correlacionando-se muitas vezes com dados clínicos e laboratoriais. No entanto, o transplante hepático e a pancreatite crônica também contribuem como causas de estenoses benignas ${ }^{2}$.

Na sequência terapêutica, a tendência é optar por meios gradualmente invasivos, assim, na indicação da drenagem biliar este princípio é válido quando reconhecemos que a solução de continuidade do peritônio parietal durante a punção transparietohepática caracteriza uma paracentese e com isso um procedimento definido como invasivo ${ }^{3}$.

A endoscopia foi considerada no início segura como um procedimento complementar diagnóstico. Contudo, com o advento de novos aparelhos e acessórios, permitiu acesso às vísceras e órgãos adjacentes ao trato gastrointestinal. Neste ponto de vista, ao conceituarmos método invasivo, definimos solução de continuidade da serosa parietal torácica ou abdominal, caracterizando, nesta situação, o padrão invasivo da endoscopia (e.g., ecopunção, neurólise do plexo celíaco, punção de gânglios torácicos e abdominais, massas mediastinais) ${ }^{3}$.

Os recursos diagnósticos não invasivos disponíveis como ultra-sonografia, tomografia computadorizada helicoidal e colangiopancreatografia por ressonância magnética, são usados genericamente para diferenciar entre estenoses malignas e benignas na tentativa de revelar a presença ou ausência de uma massa tumoral, metástase e invasão ganglionar. No entanto, tais exames avaliam apenas o nível de obstrução biliar sem revelar a etiologia da estenose, tornando-se às vezes insuficiente para certeza diagnóstica e decisão terapêutica ${ }^{3}$.

Trabalho realizado no

1. Professor Livre - Docente pela Universidade de São Paulo (USP); 2. Pós- Graduando do Departamento de Cirurgia do HCFMUSP; 3. Professor Livre-Docente pela Universidade de São Paulo (USP). 
Tabela 1 - Causas de estenoses benignas.

Pós-operatório, incluindo transplante hepático

Colangite esclerosante

Primário

Secundário

Doenças pancreáticas

Pancreatite aguda

Pancreatite crônica

Pseudocisto

Infecção / infestação

Colangite recorrente

SIDA

Parasitas

Congênitas

Outros: trauma abdominal, hemangioma, radioterapia, sarcoidose, doença hepática policística

Adaptado de Gibbons, $\mathrm{JC}^{1}$

Em estudo com 44 pacientes, a colangiorressonância detectou o nível de estenose em 96\% apresentando excelente correlação com a colangiopancreatografia retrógrada endoscópica (CPRE). Contudo, a localização precisa da lesão de acordo com a classificação de Bismuth foi de $74 \%$ e observou-se uma moderada concordância entre colangiorressonância e CPRE para avaliação do tratamento cirúrgico, com sensibilidade de $84 \%$ e especificidade de $63 \%{ }^{4}$.

\subsubsection{Estenose biliar pós-operatória}

A lesão iatrogênica do ducto biliar é a causa mais comum das estenoses benignas. Ocorre freqüentemente após colecistectomias, nas cirurgias abertas de 0,1-0,5\% e nas laparoscópicas de 0,25-1\%. Embora os traumas cirúrgicos diretos sejam os responsáveis pela maioria das estenoses, outras possíveis causas são as lesões isquêmicas microvasculares após dissecção cirúrgica e inflamação adjacente ou uso de cauterização monopolar para hemostasia. Os sintomas clínicos são icterícia, dor epigástrica, calafrio e febre. A apresentação clínica depende do tipo de lesão e é dividida em dois grupos. Nos pacientes com fístula biliar sem dreno abdominal, a bile acumula no espaço subhepático formando coleção (bilioma) ou abscesso. Nesses pacientes sinais de sepse podem ocorrer. Geralmente, icterícia não é observada nesses pacientes porque colestase não está presente. No segundo grupo, pacientes com estenose biliar a icterícia é sinal clínico dominante'. Os traumas operatórios são reconhecidos precocemente em $25 \%$ dos casos, mas podem ser descobertas até 20 anos após o procedimento inicial ${ }^{5}$. A radiofreqüência utilizada em cirurgias hepáticas contribui com $1 \%$ das lesões detectáveis.

As estenoses pós-operatórias são classificadas por Bismuth-Strasberg (Figura 1): o tipo A correspondendo a extravazamento de bile do ducto menor em continuidade do ducto hepático comum; tipo $B$, divisão e clipagem do segmento do ducto hepático direito; tipo $C$, estravasamento de bile do segmento ducto hepático direito, sem comuni-
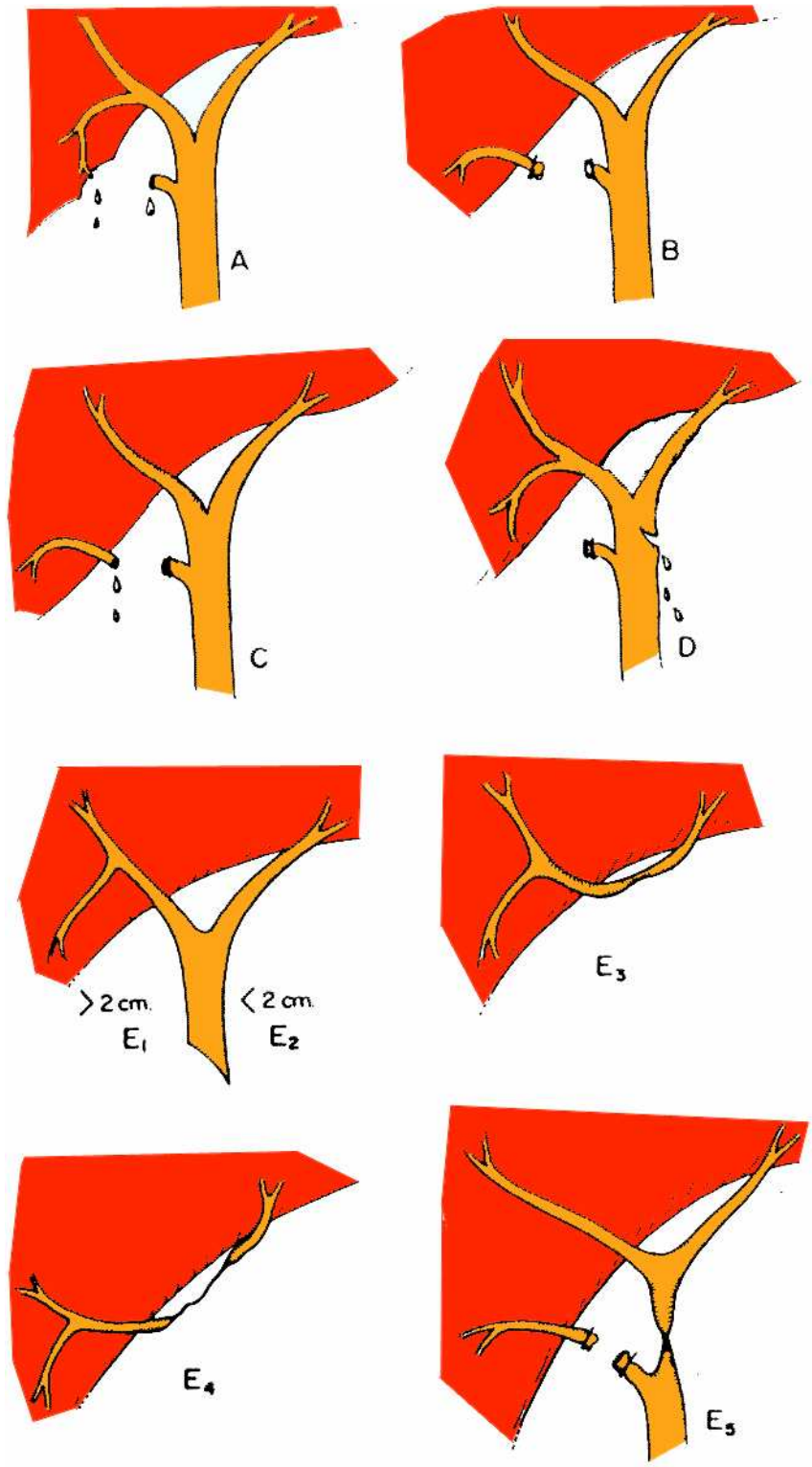

Figura 1 - Esquema da Classificação de Bismuth-Strasberg.

cação com ducto hepático comum; tipo $D$, lesão lateral do ducto hepático comum; tipo E1, lesão circunferencial do ducto comum mais de $2 \mathrm{~cm}$ da bifurcação; tipo E2, lesão circuferencial do ducto hepático comum menos de $2 \mathrm{~cm}$ da bifurcação; tipo E3, lesão circuferencial no ducto hepático comum na bifurcação; tipo E4, estenose do sistema ductal hepático direito ou esquerdo; tipo $\mathrm{E} 5$, lesão combinada do ducto principal na bifurcação e segmento do ducto hepático direito. Outra classificação corresponde a BismuthBlumgart onde a lesão distante da confluência dos hepáticos caracteriza o tipo l; a lesão da confluência preservando a junção angular superior, o tipo Il; lesão completa da confluência, o tipo III; e lesão da confluência e dos ductos hepáticos, tipo IV ${ }^{6}$.

As estenoses pós-operatórias mais freqüentes estão localizadas abaixo do hilo. O tratamento cirúrgico com sucesso é de $73-90 \%$. A morbidade varia de 7 a $26 \%$ e a mortalidade de 0 a $13 \%$; a mortalidade maior tem sido relatada em pacientes com hipertensão portal. As estenoses 
recorrentes variam de $10-35 \%$ e estão associados a fatores como tratamento cirúrgico prévio, cirrose, hipertensão portal, fístula biliar e idade avançada³.

O tratamento endoscópico inclui dilatação radial com cateteres balonados, colocação de próteses após estenose que devem ser trocadas de maneira gradual, tendo a permanecia mínima de três meses quando ocorrerá cicatrização estável, levando a uma menor probabilidade de reestenoses deste segmento. A programação é de colocação de múltiplas próteses plásticas, de 2 a 4 e com manutenção de 6 a 12 meses. O controle deverá ser feito com seis meses a um ano através de colangiorresonância ou colangiografia endoscópica. Trata-se de um procedimento menos invasivo em relação ao procedimento cirúrgico ${ }^{7}$. O índice de sucesso está em torno de $70-80 \%$, similar ao tratamento cirúrgico. Entretanto, é necessário um acompanhamento em longo prazo devido à ocorrência de complicações tardias e a possibilidade de lesão irreversível quando o tratamento não for realizado em tempo hábil. Geralmente, 80-90\% das estenoses biliares podem ser tratadas inicialmente por endoscopia ${ }^{7}$. As características preditivas de falha são estenoses acima do hilo, estenoses longas, apresentação pós-cirúrgica tardia (>três meses), variações anatômicas do trato gastrointestinal alto ou das vias biliares e condições clínicas gerais afetando a segurança da sedação prolongada ou anestesia geral ${ }^{7}$.

Por muitos anos a prótese metálica auto-expansível era indicada apenas para tratamento definitivo (paliação de tumores irressecáveis na junção bilio-pancreática), mas recentemente, esse paradigma tem sido reavaliado para doenças benignas, com uso de prótese parcial ou totalmente recobertas, que são potencialmente removíveis ${ }^{8}$.

Kahaleh et al. ${ }^{9}$ relataram que o uso de prótese metálica auto-expansível para estenose biliar benigna é possível, porém bastante controverso devido a fatores como: (1) calibre, geralmente fino, da via biliar (2) estenose anelar, impedindo boa ancoragem da prótese, favorecendo a migração (14\%) e sua localização Bismuth III e IV que por razões anatômicas prejudicariam a drenagem da via biliar contra-lateral. O uso dessas próteses em estenoses pós-transplante hepático teve melhores resultados devido à diferença de calibre da via biliar, com mais espaço para acomodar a prótese. Porém deve ser recomendado com cautela devido alto risco de perfuração.

Artifon et al. ${ }^{10}$ publicaram, em 2009, estudo comparativo do uso de prótese metálica autoexpansível recoberta por material siliconizado vs passagem de múltiplas próteses plásticas em pacientes com lesão biliar póscirúrgica classificadas em Bismuth -Blumgart I (lesão além de $2 \mathrm{~cm}$ da confluência dos ductos hepáticos). Verificou-se que a capacidade de recanalização biliar sustentada e custo foram significativamente favoráveis ao grupo prótese metálica. Neste, destaca-se também, que a migração da prótese foi significativamente menor.

\subsubsection{Lesões biliares no transplante hepático}

As complicações biliares após o transplante hepático variam de 7 a 34\%. Estas complicações incluem fístula biliar, estenoses de anastomoses, estenoses difusas, coledocolitíase e colangite. A estenose da anastomose é geralmente secundária a técnica cirúrgica, ao passo que estenoses difusas da árvore biliar extrahepática são devido à isquemia. A estenose intra e extrahepática pode também ser secundária a isquemia arterial, associada à trombose da artéria hepática. Outros fatores incluem prolongado tempo de isquemia fria, incompatibilidade do grupo $A B O$, infecção pelo CMV e rejeição.

Rerknimitr et al. ${ }^{11}$ estudaram retrospectivamente 367 pacientes submetidos a transplante hepático ortotópico com anastomose colédoco-coledociana e observou 24,5\% de complicações biliares. As principais foram: estenose $(45,5 \%)$, coledocolitíase $(30,5 \%)$ e fístula biliar (18,1\%). A terapêutica endoscópica obteve sucesso em todos pacientes com litíase e fístular biliar. Nos casos com estenose, a maioria encontrava-se no local da anastomose (78,2\%) e o restante no ducto do doador. A dilatação com balão ou cateter dilatador seguida de próteses com trocas em média de 3 - 5 meses foram realizados com sucesso em $91 \%$ dos pacientes. As complicações da colangiografia endoscópica ocorreram em 8 pacientes e compreenderam: hemorragia, perfuração causada pelo fio guia ao nível da estenose, pancreatite e migração da prótese plástica. O estudo sugere que a colangiografia endoscópica identifica as anormalidades da árvore biliar e proporciona múltiplas opções terapêuticas.

Eckhoff et al. ${ }^{12}$ verificaram a ineficácia da CPRE na avaliação e manejo da elevação assintomática de enzimas hepáticas em pacientes submetidos a transplante hepático, sendo a biópsia hepática o método invasivo ideal nesses casos. Esta conclusão ocorreu após constatarem 96\% de colangiografias normais em estudo retrospectivo de 400 pacientes.

Shah et al. ${ }^{13}$ avaliaram colangiografia endoscópica de rotina no acompanhamento pós-transplante hepático para o diagnóstico de complicações biliares. Este exame foi realizado em 89 dos 171 pacientes transplantados após 14 dias do procedimento cirúrgico. No subgrupo de pacientes com alterações das enzimas hepáticas ou suspeita de sepse, a colangiografia endoscópica foi positiva em $23 \%$ dos casos e apenas 3\% nos pacientes pós-transplante sem alterações enzimáticas. A sensibilidade para detecção das complicações biliares foi de $53 \%$ e especificidade de $98 \%$. Assim, o autor sugere que a colangiografia endoscópica deveria ser realizada em pacientes sintomáticos.

\subsubsection{Colangite esclerosante primária (CEP)}

A colangiografia endoscópica é o exame de escolha para o diagnóstico de CEP. ${ }^{14}$ Contudo, é importante reconhecer o risco de colonização bacteriana após injeção de contraste na árvore biliar parcialmente obstruída. Assim, a profilaxia antibiótica é necessária em todos os casos quando se objetiva evitar a sepse biliar, porém a drenagem biliar é de fundamental importância. A realização de escovados e/ou biópsias de qualquer estenose é importante para avaliar a presença de colangiocarcinoma. Em pacientes com CEP deve-se ter o cuidado de avaliar com detalhe as áreas 
de estreitamento e irregularidades, pois estas regiões são passiveis de serem confundidas com colangiocarcinoma.

Stiehl et al. ${ }^{15}$ prospectivamente avaliaram 106 pacientes tratados com ácido ursodeoxicólico por mais de 13 anos e observaram o desenvolvimento de estenoses. Nesse período foram diagnosticados 52 pacientes com estenose biliar, sendo que em cinco pacientes foi necessário além da dilatação endoscópica, passagem de prótese para manter drenagem da via biliar. As complicações observadas na CPRE foram: pancreatite $(5,2 \%)$, colangite $(3,3 \%)$ e perfuração do ducto biliar $(0,5 \%)$. Este estudo conclui que o tratamento endoscópico permite postergar a conduta cirúrgica, porém com a evolução da afecção, o transplante hepático torna-se a única opção de cura.

A contribuição da endoscopia biliopancreática no tratamento das deformidades biliares ocorridas na CEP inclui a drenagem através de próteses plásticas, bem como a coleta de material através de escovado citológico e biópsias com fórceps. A indicação de papilotomia deverá ser criteriosa em vista da ocorrência do refluxo duodeno biliar e suas conseqüências em longo prazo tal como colangite repetitiva, colangioesclerose e câncer biliar ${ }^{16}$.

\section{Pancreatite crônica}

A estenose do ducto biliar comum está presente em $45 \%$ dos pacientes com pancreatite crônica e em muitos casos são assintomáticos. O maior temor da pancreatopatia crônica é a fibrose parenquimatosa cefálica levando a estenose circunferencial e regular da porção intrapancreática do colédoco. A manifestação inicial é caracterizada por icterícia e dor abdominal, nestas condições já significando tradução clínica de estenose acentuada ${ }^{17}$.

Pacientes com pancreatopatia crônica ocasionalmente apresentam hepatopatia associada e esta leva ao cirurgião ter certa ressalva em uma conduta mais intervencionista. Este fato transfere ao endoscopista conduzir o tratamento inicial optando-se, na maioria das vezes, pela drenagem biliar com passagem de prótese plástica calibrosa pela estenose, porém em mais de 50\% dos casos ocorre recidiva da estenose. Por isso, a anastomose cirúrgica bilio-entérica ainda é considerada o melhor tratamento definitivo nessa doença ${ }^{18}$.

Catalano et al. ${ }^{19}$ compararam um grupo de pacientes com pancreatite crônica e estenose biliar tratado com prótese plástica biliar e outro grupo com várias próteses (4 - 5 próteses), sendo o último grupo superior por apresentar melhora mais rápida dos valores laboratoriais, aumento do diâmetro da estenose e maior benefício a longo prazo.

\section{FISTULA BILIAR}

\subsection{Etiologia e diagnóstico}

A solução de continuidade biliar é complicação grave e que necessita de avaliação racional e rigorosa de equipe multidisciplinar para determinação objetiva e segura do procedimento terapêutico de escolha. Dentre as principais causas desta afecção inclui-se os procedimentos cirúrgicos e trauma na incidência de $67 \%$ e $19 \%$, respectivamente ${ }^{20}$. Algumas características são propostas, a saber: em relação ao tempo de evolução - agudas e crônicas; em relação ao mecanismo de trauma térmica ou indireta e traumática ou direta; em relação a drenagem - interna e externa; de acordo com o débito da drenagem - débito alto, médio e baixo.

A lesão biliar intra-operatória deverá ser lembrada na ocorrência de dificuldade técnica, processo inflamatório intenso local, reoperações e pacientes com alterações anatômicas. O diagnóstico pode ser intra-operatória mediante visibilização de bile no campo cirúrgico e por meio de colangiografia intraoperatória. No entanto, a maioria dos pacientes é diagnosticada no pós-operatório através de clínica inespecífica como febrícula, dor em hipocôndrio direito e icterícia leve e sazonal. A partir destes dados, utilizamos seqüencialmente a ultra-sonografia transabdominal, tomografia helicoidal com duplo contraste e colangiorresonância quando houver programação cirúrgica ou CPRE com intenção terapêutica endoscópica. Os achados imagenológicos podem caracterizar o nível e extensão da lesão, bem como dados anatômicos da via biliopancreática ${ }^{20}$.

Rieger et $a^{21}$ referem ocorrência de $75 \%$ das fístulas biliares na porção proximal da via biliar, no entanto, as de maior dificuldade diagnóstica encontramse na porção distal.

\subsection{Tratamento endoscópico}

A escolha do procedimento endoscópico deverá ser racional e estabelecida por critérios bem selecionados como presença de fístulas de baixo (menor que $500 \mathrm{ml} /$ dia) e médio (500 a $1000 \mathrm{ml} /$ dia) débitos, curta extensão e localizadas nas porções média e distal da via biliar extrahepática. Os pacientes que não participam destas características, ou seja, fístulas de alto débito, peritonite biliar e pacientes jovens devem ser preferencialmente levadas ao tratamento cirúrgico. (Tabela 2)

O procedimento endoscópico consiste na papilotomia biliar ampla objetivando drenagem biliar fácil

Tabela 2 - Critérios de opção terapêutica da fístula biliar.

\section{Endoscópica}

Cirúrgico

Fístula de médio e baixo débito

Lesão de curta extensão

Coleção pequena e localizada

Localização biliar média e distal

Idosos e/ou comorbidades associadas
Fístulas refratárias e de alto débito

Coleções múltiplas e loculadas

Peritonite biliar

Lesões extensas e múltiplas

Jovens 
e passagem de prótese plástica com intuito de, por capilaridade, direcionar o fluxo em direção distal, diminuindo o débito fistular. A prótese atua também como fator de perviedade transpapilar, prevenindo o processo inflamatório pela ação eletrotérmica após a secção papilar²2.

Cotton et al. ${ }^{23}$ referem sucesso de $87 \%$ no tratamento endoscópico em 39 pacientes com lesão biliar operatória seguida de fístula.

No Serviço de Endoscopia Digestiva do HCFMUSP, realizamos papilotomia associada à passagem de endoprótese biliar plástica. A prótese de escolha varia em extensão, no entanto, damos preferência às mais calibrosas e de poliuretano. A retirada, habitualmente, fazse na sala de radiologia após 10 a 15 dias. Lembramos que a indicação do tratamento endoscópico deve ser criteriosa, realizada por endoscopista experiente e em centros que forneçam recursos adequados para manipulação biliopancreática.

\section{ESTENOSES MALIGNAS}

\subsection{Etiologia e diagnóstico}

A estenose maligna das vias biliares pode originar-se a partir do epitélio biliar ou de vísceras e gânglios adjacentes. Quando a origem neoplásica for epitelial intra ou extrahepática caracteriza-se tumor primário, e quando de origem não epitelial, secundário, sendo incluído neste caso o câncer de vesícula biliar ${ }^{24}$.

A maioria dos pacientes com câncer biliopancreático é diagnosticada em fase avançada, com o aparecimento de icterícia obstrutiva, e sem possibilidade de tratamento cirúrgico curativo. Nestas condições, a paliação endoscópica é considerada opção tanto na drenagem biliar, antecipando a operação paliativa ou potencialmente curativa, ou mesmo definitivo na ausência de condições cirúrgicas ${ }^{25}$.

A biópsia percutânea dirigida radiologicamente é freqüentemente difícil, especialmente em colangiocarcinoma, e possui um risco pequeno de implante no trajeto. A citologia exfoliativa obtida pela CPRE e colangiografia percutânea apresenta uma alta taxa de falso negativo. Contudo, Mohandas et al. ${ }^{26}$ relataram um aumento da sensibilidade na identificação citológica na bile em amostras obtidas após dilatação da estenose (63.3 vs $26.6 \%$ do grupo controle). A citologia do escovado na CPRE tem sensibilidade de $70 \%$ e especificidade de $100 \%$, mas geralmente esta técnica tem sido desapontadora, pois a maioria dos estudos relata uma sensibilidade entre 40 e $50 \%$.

A biópsia endobiliar tem sido comparada com a citologia com escovado em vários estudos. Em um estudo com 223 pacientes, Ponchon et al..$^{27}$ obteve material adequado em $90 \%$ com escovado comparado a $55 \%$ da biópsia; e ambos obtiveram mais sucesso quando o nível da estenose acometia o ducto biliar comum. Não houve diferença significativa da sensibilidade dos métodos. Contudo, quando usado em combinação, houve uma melhora significativa da sensibilidade (35\% com escovado vs $43 \%$ biopsia vs $63 \%$ combinado). Assim, uma combinação de técnicas é recomendada para maior sensibilidade diagnóstica.

\subsubsection{Neoplasia pancreática}

O adenocarcinoma da cabeça do pâncreas contribui com 40 a $60 \%$ das neoplasias do confluente duodenobilio-pancreático e caracteriza-se pela clínica de icterícia, dor e emagrecimento na maioria dos pacientes. O diagnóstico imagenológico permite o melhor direcionamento no estadio, cabendo a ecoendoscopia setorial a punção diagnóstica para definição citopatológica e imunohistoquímica, e à CPRE o tratamento paliativo através da passagens de próteses plásticas ou metálicas autoexpansíveis ${ }^{28}$.

Nos casos irressecáveis torna-se mínimo o benefício gerado pelo tratamento cirúrgico, exceto quando houver obstrução duodenal. Além disso, a icterícia pode ser paliada por procedimentos menos invasivos que o ato operatório, como passagens de próteses percutâneas ou por via endoscópica ${ }^{29}$.

Artifon et al. ${ }^{30}$ descreveram estudo de importante impacto médico e sócio-econômico no qual foi comparada a prótese metálica biliar passada por endoscopia versus a operação derivativa em pacientes com câncer avançado de cabeça de pâncreas. Neste estudo, a qualidade de vida avaliada pelo índice SF-36 e custo total do procedimento foram favoráveis à paliação endoscópica.

Com intuito de se obter um método minimamante invasivo na paliação do câncer de pâncreas, Artifon et al. ${ }^{31}$ descreveram a combinação da laparoscopia com a endoscopia. A técnica é demonstrada pela seqüência de figuras abaixo (Figuras 2,3 e 4) e trata-se de passagem do sistema de prótese metálica auto-expansível por trocarter laparoscópico subcostal de $5 \mathrm{~mm}$ e com acesso anterógrado por meio de secção puntiforme do ducto hepático comum (Figura 3) e/ou ducto cístico (Figura 2). O papel da duodenoscopia nesse estudo foi apenas para verificação do posicionamento transpapilar da prótese metálica, podendo ser dispensável durante a evolução da curva de aprendizado da equipe cirúrgica.

\subsubsection{Colangiocarcinoma}

É considerada a neoplasia primária mais comum da via biliar. Apresenta incidência gradualmente maior em direção proximal no hilo hepático. A colangiografia caracteriza-se por estreitamento irregular e segmentar que não modifica a direção do eixo biliar. Este detalhe é importante ser lembrado quando o estreitamento neoplásico deve ser diferenciado do câncer de cabeça de pâncreas, pois neste observamos desvio do eixo biliar, parada de progressão do contraste no ducto pancreático e paralelismo dos ductos biliar e pancreático, caracterizando o "sinal do duplo canal".

A CPRE, diferente de outros métodos, possibilita melhor diagnóstico citopatológico através do escovado e biópsias biliares. Deve-se optar, habitualmente, pelas duas 


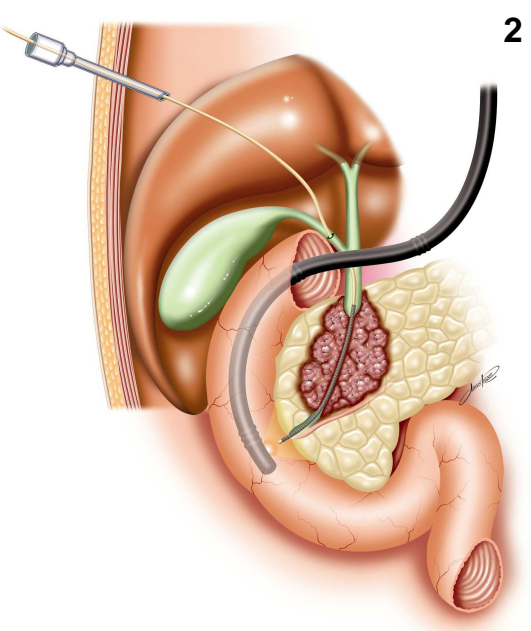

2

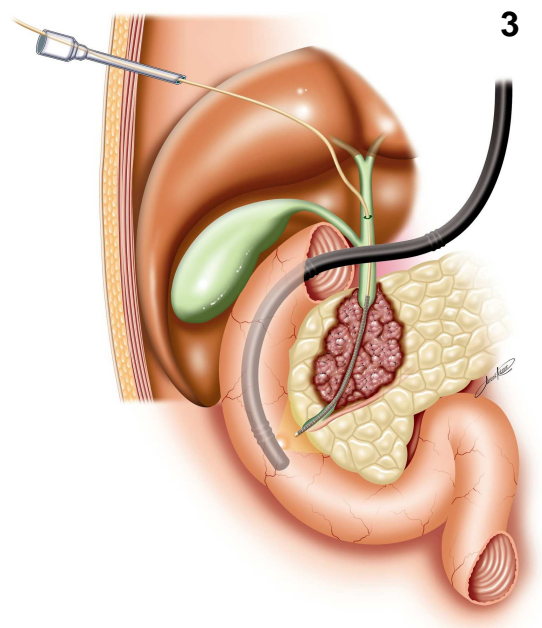

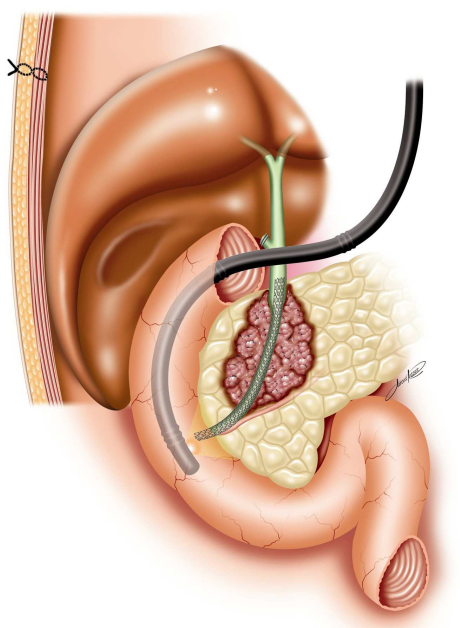

Figuras 2, 3 e 4 - Imagem artística da passagem de prótese metálica pelo ducto cístico (2), ducto hepático comum (3) e prótese posicionada na posição transtumoral (4).

táticas de obtenção de material para análise, pois a sensibilidade chega a $54 \% .32$.

\subsubsection{Câncer de vesícula}

Ocorre em 10 a 15\% das neoplasias biliares e depende em grande parte de achados anatomopatológicos durante exame macroscópico da peça cirúrgica. Nos casos precoces a colecistectomia é terapêutica, porém o habitual é encontrarmos pacientes com estádio avançado, nos quais a paliação é possível através de passagem de prótese plástica ou metálica por endoscopia. Em 20 a 35\% dos pacientes a infiltração peritumoral caracteriza invasão e particulariza achado colangiográfico de falha irregular, ocupando lateralmente a via biliar extrahepática proximal. Deviere et al. ${ }^{33}$ alertam quanto ao cuidado que se deve ter quando, durante a colangiografia, se visibilizar vesícula exclusa. Destes pacientes 3 a 6\% podem apresentar neoplasia de vesícula biliar, quando somados outros fatores como tabagismo, idade acima de 60 anos e história familiar.

\subsubsection{Linfonodomegalia hilar secundária}

O hilo hepático e retroperitônio são ricos em linfonodos e na ocorrência de afecções sistêmicas como linfoma não Hodgkin, leucemia linfóide crônica e metástases neoplásicas, estes linfonodos aumentam em até 10 vezes de diâmetro, determinado após coalescerem, massa sólida hilar capaz de levar a estreitamento irregular em qualquer nível da via biliar extrahepática. Nesta situação a CPRE é o tratamento na drenagem biliar temporária ${ }^{34}$.

\subsubsection{Câncer de papila}

A neoplasia papilar diferencia-se das outras neoplasias do confluente duodeno-bilio-pancreático por determinar o aparecimento precoce de icterícia e dor abdominal, coincidindo temporalmente com tumor em estádio precoce (T1NOM0). Estes comemorativos clínicos permitem ao gastroenterologista experiente complementar objetivamente a investigação, utilizando-se precisamente a duodenoscopia com biópsia e métodos imagenológicos para estadiamento locorregional e a distância ${ }^{35}$.
O tratamento cirúrgico é o padrão ouro e tem na gastroduodenopancreatectomia a possibilidade de cura oncológica de 70 a $90 \%$ dos pacientes, dependendo do estágio da doença ${ }^{35}$.

A opção endoscópica é útil em pacientes com lesões potencialmente malignas como adenoma de papila com displasia, através da ampulectomia. Nos casos de pacientes sem condições clínicas que não devem ser submetidos à lapatoromia ou nos estágios avançados da doença, a paliação pode ser obtida com uso de próteses plástica ou metálica ${ }^{35}$.

\section{hilar avançada}

\subsection{Tratamento endoscópico da neoplasia}

O processo de diagnóstico e tratamento do tumor de Klatskin deverá ser tomado por equipe multidisciplinar, cabendo a responsabilidade ao oncologista, cirurgião, radiologista e endoscopista bilio-pancreático. A acurácia diagnóstica, estadiamento do tumor e tipo histológico deverá ser obtida pela equipe multidisciplinar através da TC helicoidal, ressonância magnética e ou ecoendoscopia com punção aspirativa. Em casos selecionados a colangiografia percutânea ou endoscópica com ultra-som intraductal, poderá ser usada.

A grande maioria dos tumores hilares primários são irressecáveis devido a extensão local, metástases ou inoperabilidade por comorbidades graves e isto leva a expectativa de sobrevida menor que $10 \%$ em cinco anos. Sendo assim, a paliação endoscópica torna-se a principal opção com o objetivo de melhorar a qualidade de vida ${ }^{32}$.

O tratamento endoscópico está relacionado a maior risco de complicações, sendo a colangite encontrada em 10 a $40 \%$ dos pacientes ${ }^{36}$. O risco de sepse biliar pode ser reduzido significativamente por meio de uma cuidadosa avaliação imagenológica da extensão intrahepática do tumor através de colangiorressonância (MRCP) e cuidados durante a colangiopancreatografia endoscópica retrógrada (CPRE) em relação a injeção do contraste e contaminação.

Uma opção de drenagem biliar combinada através da punção hepática percutânea e endoscopia é a téc- 
nica de "Rendez-Vous". Neste procedimento, a obtenção do acesso biliar é feita pela punção transparietohepática, onde o fio guia é direcionado anterogradamente através da papila e recolhido para o canal de trabalho do duodenoscópio. Tal procedimento é de escolha em neoplasias hilares avançadas onde não houve contrastação proximal durante a colangiografia retrógrada ${ }^{37}$.

Entre as próteses plásticas preferem-se as de maior diâmetro (10 a 12 French), porém a dificuldade de passagem pelo estreitamento, mesmo após dilatação retrógrada e progressiva, leva a uso da opção de prótese de menor diâmetro como de 8,5 Fr. Estas próteses são habitualmente de polietileno ou poliuretano, apresentam diferentes tipos de curvaturas e sistemas de ancoragem lateral. Porém, em vários ensaios clínicos, não se observam diferenças significantes que possam alterar o sucesso clínico e técnico final ${ }^{38,39}$.

A passagem única de próteses plásticas de até 10 French é possível sem papilotomia, ou apenas com dilatação balonada da papila maior, porém quando mais de uma prótese for necessária a papilotomia será mandatória.

De Palma et al. ${ }^{36}$ referem que a drenagem unilateral apresenta melhor sucesso técnico e menores complicações em relação à bilateral. Acreditamos que a opção de passagem de uma ou mais próteses deva ser ordenada por fatores como o tipo e extensão do estreitamento, número de segmentos hepáticos opacificados e possibilidade de acesso para drenagem, experiência e habilidade do endoscopista.

Alguns estudos comparando a prótese plástica com a auto-expansível metálica para tratamento paliativo do tumor de hilo hepático mostraram índices significativamente menores de obstrução e colangite a favor da prótese metálica ${ }^{40}$. A prótese metálica é passada com certa facilidade, pois o sistema é composto por cateteres de 7,5 ou 8 French e poderá ser posicionado pelo estreitamento com ou sem dilatação retrógrada progressiva. Os sistemas são pouco variáveis em relação a itens como grau de opacidade, grau de flexibilidade do material e mecanismo de liberação, porém a escolha é determinada pela facilidade de uso e experiência do endoscopista com o material.

Cheng et al. ${ }^{41}$ estudaram retrospectivamente a utilização de prótese metálica uni ou bilateral em 36 pacientes com tumor de Klatskin onde sete pacientes foram classificados como Bismuth II, 13 tipo III e 16 tipo IV. Em 22 (61\%) pacientes foram passadas uma prótese, 9 (25\%) bilateral, 1 (3 \%) com três próteses e em quatro (1 1 \%) pacientes houve passagem de duas próteses unilaterais. $\mathrm{O}$ sucesso técnico foi de $97 \%$, a morbidade e mortalidade precoce foi 14 e $6 \%$, respectivamente. Neste estudo, o tratamento teve caráter paliativo definitivo em $69 \%$ dos pacientes.

Acreditamos que a prótese metálica apresenta melhor relação custo benefício, pois reduz a necessidade de repetidas endoscopias, facilidade técnica e baixo índice de complicações como colangite e obstrução.

O objetivo da drenagem biliar não é somente aliviar a icterícia, mas também diminuir a colestase, para isto é importante a drenagem completa dos segmentos hepáticos contrastados e represados. Variações envolvendo o ducto hepático direito são freqüentes e ocorrem em $40 \%$ da população e podem interferir em detalhes técnicos no acesso ao segmento obstruído. Quando múltiplos ductos estão tomados, o posicionamento único da prótese em um dos ramos pérvios leva a drenagem de apenas 30\% do fluxo biliar, portanto haverá discreta redução da icterícia sem aliviar a colestase. Nesta situação o risco de colangite estará relacionado com a área contrastada tendo a possibilidade de drenagem. Em vista disto o número de próteses é proporcional ao de ramos obstruídos.

Alguns autores recomendam a drenagem lobar única, porém não há publicações comparando drenagem incompleta e completa, mas somente unilateral com bilateral. Sendo assim um paciente classificado como Bismuth III e IV necessitariam de mais de duas próteses para determinar uma ótima drenagem biliar ${ }^{26,34}$.

Hintze et al. ${ }^{42}$ referem que o uso de colangiopancreatografia por ressonância magnética no estudo pré CPRE possibilitaria identificar qual o melhor segmento para drenagem após avaliação imagenológica dos ductos bilares. Com isso houve melhor seleção na injeção de contraste e melhor posicionamento da prótese no ducto selecionado, diminuindo assim o risco de colangite para $6 \%$ além de não utilização de antibióticos, conseguindo neste estudo mortalidade nula.

Nos casos de tumores irressecáveis, a amostra tecidual e o planejamento quimioterápico devem ser obtidos através da CPRE e por ecopunção. As drenagens paliativas, através de "bypass" cirúrgico ou por prótese, deveriam ser realizadas de acordo com a experiência da instituição. Contudo, nos casos de CPRE com passagem de prótese preferimos endoprótese metálica em pacientes com expectativa de vida maior que seis meses, pois permanece patente por tempo maior que as próteses plásticas ${ }^{43}$.

\section{ASPECTOS TÉCNICOS}

Após a canulação profunda e seletiva da via biliar, a papilotomia é usualmente realizada, especialmente se mais de uma prótese for necessária. A dilatação balonada papilar ou óstio papilar permite passagem única de prótese sem necessidade de papilotomia, incluindo-se também pacientes com coagulopatia. O fio guia teflonado deve atingir a árvore intrahepatica, permitindo a seqüência: dilatação da estenose, escovado citológico e instalação da prótese. A preferência inicial é por fios hidrofílicos, devido à alta eficácia em transpor estreitamentos graves. A dilatação pode ser progressiva e gradual utilizando-se cateteres dilatadores ou dilatação balonada radial, facilitando a passagem de endopróteses plásticas, não-expansíveis.

Atualmente dois tipos básicos de próteses estão disponíveis: as plásticas, de menor custo e obstrução mais precoce e as metálicas, auto expansíveis, de maior custo unitário. As próteses auto expansíveis têm maior diâmetro e conseqüentemente menor chance de obstrução, permi- 
tem procedimento único, custo relativo menor, menor tempo de internação, menor número de exames subseqüentes e baixos índices de complicações como migração e colangite. As próteses plásticas são preferíveis quando a sobrevida esperada for de seis meses, enquanto as auto-expansíveis são indicadas quando a perspectiva for maior que seis meses de sobrevida.

Em situações de obstrução biliar complexa, com necessidade de passagem de mais de uma prótese, é preferível iniciar pelo ducto hepático esquerdo seguido pelo direito. Quando utilizamos mais de uma prótese auto expansível, é mandatário passar os fios guias (2 ou 3) para passagem e liberação seqüencial das próteses ${ }^{40}$.

Em centros de referência, o sucesso da passagem de próteses é de aproximadamente $90 \%$. O sucesso do alívio do prurido e diminuição da icterícia nos pacientes com drenagem completa está em torno de $80 \%^{37}$.

Os níveis aumentados da bilirrubina $(14 \mathrm{mg} / \mathrm{dl})$ desencadeiam disfunções renais, pulmonares, neurológicas, cardíacas e até mesmo coagulopatia. O ato operatório realizado nestas condições ocasionaria alta morbimortalidade, no entanto com drenagem biliar endoscópica pré-operatória ocorrerá diminuição dos níveis de bilirrubina e conseqüentemente melhores condições para o ato operatório.

Em relação a qualidade de vida, existem trabaIhos demonstrando que após o primeiro mês da drenagem biliar endoscópica com diminuição dos níveis de bilirrubina, há significante melhora da função social e psicológica ${ }^{40}$.

As próteses plásticas são efetivas para a drenagem em curto prazo, fáceis de inserção, porém obstruem em três a cinco meses. A obstrução ocorre pela contaminação bacteriana, deposição de biofilme na superfície interna das próteses e subseqüente acúmulo de barro biliar, constituindo seu maior defeito. Próteses locadas para obstrução hilar parecem ocluir mais rapidamente que aquelas posicionadas em obstruções distais.

A prótese ideal e com ótimo fluxo seria caracterizada por baixo custo, extensão curta, diâmetro maior, longa patência e de fácil inserção. Lembramos que a efetividade do fluxo depende diretamente do diâmetro da prótese, e não do número de próteses.

Alguns estudos comparando próteses plásticas e metálicas não mostram diferença em termos de alívio de icterícia, influência preoperatória e sobrevida, no entanto, internação hospitalar, repetidas CPRE e maior patência, levam a um custo final relativamente menor e com melhor qualidade de vida proporcionada pela prótese autoexpansível ${ }^{7,9,39}$.

Kaassis et al. ${ }^{44}$ referem que presença de metástase hepática é preditiva de sobrevida e delineiam o uso racional da prótese plástica ou auto expansível. Kim et al. ${ }^{40}$ relatam que $70 \%$ do diâmetro máximo expandido ocorre nas primeiras 24 horas e independe de fatores relacionados a natureza da neoplasia.

\section{CONSIDERAÇÕES FINAIS}

Frente a um estreitamento biliar, devemos dispor de equipe multidisciplinar experiente em afecções biliopancreáticas, além de recursos imagenológicos capazes de nortear a melhor conduta terapêutica curativa ou paliativa.

Nas estenoses benignas a identificação do nível da lesão e caracterização anatomopatológica são fundamentais para o planejamento cirúrgico.

Nas estenoses malignas o estadiamento locorregional, bem como a coleta de citomaterial se faz pela ecoendoscopia setorial. Os métodos imagenológicos participam no rastreamento de gânglios e metástases a distância, assim permitem a obtenção de adequado estadiamento oncológico para definir a intenção de tratamento.

A escolha da paliação deverá ser tomada em consenso entre o cirurgião, oncologista, endoscopista e familiares.

\title{
A B S T T R A C T
}

\begin{abstract}
Standard surgical techniques offer a good chance of cure for the majority of patients affected by extrahepatic benign biliary stricture. Nevertheless, operative repair has a long-term recurrence rate of stricture in $10 \%$ to $30 \%$ of patients. Advances in endoscopic procedures have provided alternative options of relieving biliary obstructions, but prolonged length of treatment and rehospitalization have to be considered if endoscopy is performed. Malignant stenoses are an important factor determining cholangitis, jaundice and pain and it's each systemic consequences. The endoscopic approach intends to create a deviation of bile juice to duodenum by means of metal or plastic stents aiming a safe palliation. The purpose of this review is to show to the readers effectiveness of endoscopic treatments of benign and malignat stenosis of the bile duct and pancreatic.
\end{abstract}

Key words: Bile duct. Extrahepatic biliary tracts. Stents.

\section{REFERÊNCIAS}

1. Yeo CJ, Lillemoe KD, Ahrendt SA. Operative management of strictures and benign obstructive disorders of the bile duct. In: Zuidema GD, Yeo CJ, Orringer MB, editors. Shackelford's surgery of the alimentary tract 5th ed. Philadelphia: WB Saunders Company. 2002: 247-61

2. Gibbons JC, Williams SJ. Progress in the endoscopic management of benign biliary strictures. J of Gastroenterol \& Hepatol. 1998;13:116-124. 
3. Hall JG, Pappas TN. Current management of biliary strictures. J Gastrointest Surg. 2004;8:1098-110.

4. Courbière M, Pilleul F, Henry L, Ponchon T, Touzet S, Valette PJ. Value of Magnetic Resonance Cholangiography in Benign and Malignant Biliary Stenosis: Comparative Study with Direct Cholangiography. J Comput Assist Tomogr. 2003;27(3):31520.

5. Gouma DJ, Obertop $\mathrm{H}$. Management of bile duct injuries: treatment and long-term results. Dig Surg. 2002;19:117-122.

6. Bismuth $\mathrm{H}$, Majno PE. Biliary strictures: classification based on the principles of surgical treatment. World J Surg. 2001;25:1241-1244.

7. Vitale GC, Tran TC, Davis BR, Vitale M, Vitale D, Larson G. Endoscopic management of postcholecystectomy bile duct strictures. J Am Coll Surg. 2008;206:918-25.

8. Familiari P, Bulajic M, Mutignani M, Lee LS, Spera G, Spada C, Tringali A, Costamagna $G$. Endoscopic removal of malfunctioning biliary selfexpandable metallic stents. Gastrointest Endosc. 2005;62:903-910.

9. Kahaleh M, Behm B, Clarke BW, Brock A, Shami VM, De La Rue SA, Sundaram V, Tokar J, Adams RB, Yeaton P. Temporary placement of covered self-expandable metal stents in benign biliary strictures: a new paradigm? (with video). Gastrointest Endosc. 2008;67:446-45.

10. Artifon ELA, Furuya C K, Kumar A, Silveira E, Sakai C, Elias M, Paione JB. A Prospective Randomized Trial and a Cost-Effectiveness Analysis of Plastic Versus Covered Self-Expandable Metal Stent in Patients with Benign Biliary Stricture. Gastrointest Endosc. 2009;69(5): 136.

11. Rerknimitr R, Sherman S, Forgel EL, Kalayci C, Lumeng L, Chalasani N, Kwo P, Lehman GA. Biliary tract complications after orthotopic liver transplantation with choledochocholedochostomy anastomosis: endoscopic findings and results of therapy. Gastrointest Endosc. 2002; 55:224-31.

12. Eckhoff DE, Baron TH, Blackard WG, Morgan DE, Crowe R, Sellers M, McGuire B, Contreras JL, Bynon JS. Role of ERCP in asymptomatic orthotopic liver transplant patients with abnormal liver enzymes. Am J Gastroenterol. 2000; 95:141- 4.

13. Shah SR, Dooley J, Agarwal R, Patch D, Burroughs AK, Rolles K, Davidson BR. Routine endoscopic retrograde cholangiography in the detection of early biliary complications after liver transplantation. Liver Transpl. 2002; 8:491-4.

14. Mendes FD, Lindor KD. Primary sclerosing cholangitis. Clin. Liver Dis. 2004; 8:195-211.

15. Stiehl A, Rudolph G, Kloters-Plachky P, Sauer P, Walker S Development of dominant bile duct stenoses in patients with primary sclerosing cholangitis treated with ursodeoxycholic acid: outcome after endoscopic treatment. J Hepatol. 2002; 36:151-6

16. Rudolph G, Gotthardt D, Klöters-Plachky P, Kulaksiz H, Rost D, Stiehl A.. Influence of dominant bile duct stenoses and biliary infections on outcome in primary sclerosing cholangitis. J Hepatol. 2009;51(1):149-55

17. Farnbacher MJ, Rabenstein T, Ell C, Hahn EG, Schneider HT. Is endoscopic drainage of the common bile duct stenoses in chronic pancreatitis up-to-date? Am J Gastroenterol. 2000;95:1466-71.

Cahen DL, van Berkel AM, Oskam D, Rauws EA, Weverling GJ, Huibregtse K, Bruno MJ. Long-term results of endoscopic drainage of common bile duct strictures in chronic pancreatitis. Eur J Gastroenterol Hepatol. 2005;17:103-8.

19. Catalano M, Linder J, George S, Alcocer E, Geenen JE. Treatment of symptomatic distal common bile duct stenosis secondary to chronic pancreatitis: comparison of single vs. multiple simultaneous stents. Gastrointest Endosc. 2004; 60: 945-52.

20. Jab $\square$ oñska B, Lampe P. latrogenic bile duct injuries: Etiology, diagnosis and management. World J Gastroenterol. 2009; 15(33): 4097-104

21. Rieger R, Sulzbacher $H$, Woisetschlager R Schrenk $P$, Wayand W. Selective use of ERCP in pacients undergoing laparoscopic cholecystectomy. World J Surg. 1994; 18:900-4.

22. Vitale GC, Tran TC, Davis BR, Vitale M, Vitale D, Larson G. Endoscopic management of postcholecystectomy bile duct strictures. J Am Coll Surg. 2008;206:918-25.
23. Cotton PB, Lehman G, Vennes J Geenen JE, Russell RC, Meyers WC, Liguory C, NickI N. Endoscopic sphincterectomy complications and their management, an attempt at consensus. Gastroenterol Endosc. 1991; 37: 383-93.

24. Van Beers BE. Diagnosis of cholangiocarcinoma. HPB (Oxford). 2008; 10(2): 87-93.

25. National comprehensive cancer network clinical practice guidelines in oncology. Hepatobiliary cancers. NCCN 2008(2). Available from: http://www.nccn.org.

26. Mohandas KM, Santhi VS. Diagnosis of malignant obstructive jaundice by bile cytology: results improved by dilating the bile duct strictures. Gastrointest Endosc. 1994; 40: 150-4.

27. Ponchon T, Gagnon P, Berger F, Labadie M, Liaras A, Chavaillon A, Bory R. Value of endobiliary brush cytology and biopsies for the diagnosis of malignant bile duct stenosis: results of a prospective study. Gastrointest Endosc. 1995; 42: 565-72.

28. Mancuso A, Calabro F, Sternberg CN. Current therapies and advances in the treatment of pancreatic cancer. Crit Rev Oncol Haematol. 2006;58:213-41.

29. Scott EN, Garcea G, Doucas H, Steward WP, Dennison AR, Berry DP. Surgical bypass vs. endoscopic stenting for pancreatic ductal adenocarcinoma. HPB (Oxford). 2009; 11(2): 118-24.

30. Artifon ELA, Sakai P, Cunha JE, Dupont A, Filho FM, Hondo FY, Ishioka S, Raju GS. Surgery or Endoscopy for paliation of biliary obstruction due to metastatic pancreatic cancer. Am J Gastroenterol. 2006;101: 2031-7.

31. Artifon ELA, Rodrigues AZ, Marques S, Halwan B, Sakai P, Bresciani C, Kumar A. Laparoscopic deployment of biliary self-expandible metal stent (SEMS) for one-step palliation in 23 patients with advanced pancreatic-biliary tumors - a pilot trial. J Gastrointestinal Surg. 2007; 11: 1686-91

32. Wiersema MJ, Lehman GA, Sherman S, Hawes RH, Earle DT. Endoscopic brush cytology, fine needle aspiration and forceps biopsy in the evaluation of malignant biliary strictures. Gastrointest Endosc. 1993; 39: A352

33. Deviere J, Baize M, de Toeuf J, Cremer M. Long-term followup of Patients with Hilar Malignant Stricture Treated by Endoscopie Intenal Biliary Drainage. Gastrointest Endosc. 1988; 34:95-101.

34. De Palma GD, Masone S, Rega M, Simeoli I, Salvatori F, Siciliano S, Maione F, Girardi V, Celiento M, Persico G. Endoscopic approach to malignant strictures at the hepatic hilum. World J Gastroenterol. 2007;13(30):4042-5.

35. Lorenzo-Zúñiga $\vee$, Moreno De Vega $V$, Domènech $E$, Boix J. Diagnosis and treatment of ampullary tumors. Gastroenterol Hepatol. 2009;32(2):101-8.

36. De Palma GD, Gailoro G, Siciliano S, lovino P, Catanzano C. Unilateral versus bilateral endoscopic hepatic duct drainage in patients with malignant hilar biliary obstruction: Results of a prospecúve, randomized and controlled study. Gastrointest Endosc. 2001; 53: 547-53.

37. Scapa E, Peer A, Witz E, Eshchar J. "Rendez-vous" procedure (RVP) for obstructive jaundice. Surg Laparosc Endosc. 1994;4(2):825.

38. Aljiffry M, Walsh MJ, Molinari M. Advances in diagnosis, treatment and palliation of cholangiocarcinoma: 1990-2009. World J Gastroenterol. 2009;15(34):4240-62.

39. Ito F, Cho CS, Rikkers LF, Weber SM. Hilar cholangiocarcinoma: current management. Ann Surg. 2009;250(2):210-8.

40. Kim HS, Lee DK, Kim HG, Park JJ, Park SH, Kim JH, Yoo BM, Roe IH, Moon YS, Myung SJ. Features or Malignant Biliary Obstruction Affecting the Patency of Metalic Stents: A Multicenter Study, Gastrointest Endosc. 2002;55:359-65.

41. Cheng WR, Kortan P. Outcome in patientes with bifurcation tumors who undergo unilateral versus bilateral hepatic duct drainage. Gastrointest Endosc. 1998; 47: 354-62.

42. Hintze RE, Abou-Rebyeh $H$, Adler A Veltzke-Schlieker W, Felix R, Wiedenmann B. Magnetic resonance cholangiopancreatographyguided unilateral endoscopic stent placement for Klatskin tumors. Gastrointest Endosc. 2001:53:40-6.

43. Beilstein MC, Kochman ML. Biliary Endoscopy. Current Opinion in Gastroenterol. 2003;19:281-7 
44. Kaassis M, Boyer J, Dumas R, Ponchon T, Coumaros D, Delcenserie R, Canard JM, Fritsch J, Rey JF, Burtin P. Plastie or Metal Stents for Malignant Stricture Of the Common Bile Duct? Results of a Randomized Prospective Study. Gastrointest Endosc. 2003;57:178-82.

Recebido em 12/11/2008

Aceito para publicação em 05/01/2009

Conflito de interesse: nenhum

Fonte de financiamento: nenhuma

\section{Como citar esse artigo:}

Artifon ELA, Couto Júnior DS, Sakai P. Tratamento endoscópico das lesões das vias biliares. Rev Col Bras Cir. [periódico na Internet] 2010; 37(2). Disponível em URL: http://www.scielo.br/rcbc

\section{Endereço para correspondência:}

Everson L. A . Artifon

E-mail: eartifon@hotmail.com 